\title{
High-Throughput Analysis in the Pharmaceutical Industry
}

\author{
"This book remains a quick and valuable resource for readers, ranging from professionals to academics, to gain knowledge \\ about the most recent developments of high-throughput analysis in the pharmaceutical industry."
}

LC-MS has become a commonly used analytical method in the pharmaceutical industry. However, the dramatic increase in the number of new chemical entities and the evershortening timelines in drug discovery have posed significant challenges to its applications in drug analysis. In order to deal with these potential bottlenecks, tremendous efforts have been taken to come up with new and innovative approaches to enhance the overall throughput of sample analysis while maintaining good selectivity and sensitivity. This book focuses on the discussion of the most recent developments in automated sample preparation, parallel LC separation and more efficient MS detection. It provides the readers with a good perspective of high-throughput drug analysis in the pharmaceutical industry.

\section{"The book has a good balance of both theoretical and practical information that should appeal to a broad base of readers."}

High-throughput analysis has become increasingly important in order to meet the significant challenges presented in the pharmaceutical industry in recent years. Such challenges arise due to advances in combinatorial chemistry, greater demands in early pharmaceutical profiling of new chemical entities and the need to closely monitor therapeutic drugs in preclinical and clinical trials with ever-shortening timelines. Tremendous efforts have been taken to develop innovative strategies and techniques to enhance the overall throughput of sample analysis with good selectivity and sensitivity still maintained. This book, edited by Perry G Wang, provides excellent up-to-date reviews on the newest developments of high-throughput analysis in drug discovery and development.

LC-MS has been established as the most commonly used analytical method in the pharmaceutical industry. Thus, this book mainly focuses on how to enhance analytical throughput by optimizing conditions to achieve fast and efficient LC separation and to improve MS productivity, together with applying automated sample preparation. The chapters in this book are put together by a panel of experienced scientists and researchers from the pharmaceutical industry, academics and instrument manufacturers. The book has a good balance of both theoretical and practical information that should appeal to a broad base of readers.

Sample preparation is a critical step in highthroughput drug analysis and can ultimately determine the quality of the results generated. The goal of sample preparation is to remove potential interferences from endogenous components in biological matrices and other molecules that may compromise the analyte detection. A thorough review of high-throughput sample preparation techniques for analyzing drugs and metabolites in biological matrices and for purifying combinatorial libraries is presented in Chapter 1. The main focus of the chapter is devoted to the theories and applications of three commonly used techniques (i.e., SPE, liquid-liquid extraction [LLE] and protein precipitation). Automated applications using these extraction techniques are also presented in Chapters 2, 7 and 10-12. It is worth noting that online SPE is of particular interest due to its high applicability to automation. Column-switching systems and turbulent-flow chromatography for online SPE are reviewed more extensively in Chapters 2, 10 and 11. In addition, information on other novel approaches such as solid-phase microextraction, solid-supported LLE, liquid-phase microextraction, molecular-imprinted polymers and restricted access media can be found in Chapters 1 and 13.

HPLC is frequently used for analyte separation in high-throughput analysis because of its great flexibility, scalability and ruggedness. However, chromatographic separation can be time consuming and, potentially, a major ratelimiting step. Theoretical aspects on how to maximize the speed of chromatographic separation without compromising peak capacity

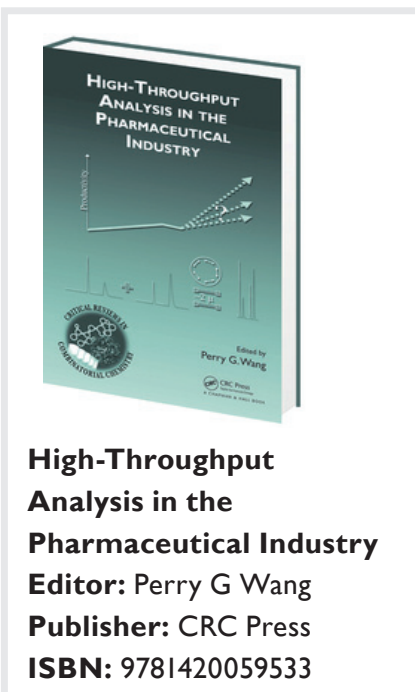

\section{Keyang Xu}

Genentech, Inc., I DNA Way, South San Francisco,

CA 94080, USA

Tel.: +I 6502254525

Fax: + I 6507424933

E-mail: xuk@gene.com

DRUg DISCOVERY

The process by which drugs are designed, synthesized, characterized and screened

Automated sample PREPARATION

Sample pretreatment process using automatic devices to remove potential interferences

\section{FUTURE SCIENCE}




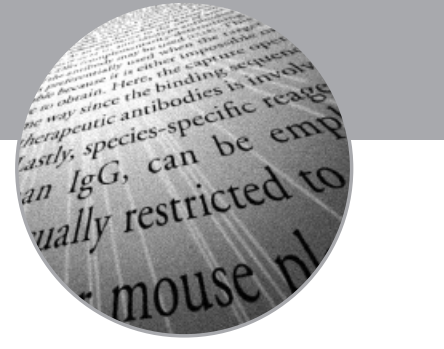

are presented in Chapters 3,13 and 14. Several major parameters such as column length, column diameter, particle size, pore size, flow rate and mobile-phase composition are discussed in these chapters. Recent developments in ultraperformance LC have improved both speed and resolution by using columns packed with sub$2-\mu \mathrm{m}$ particles. Reviews on ultraperformance LC are presented in Chapters 7, 9 and 11-13. Advances in capillary- and nano-HPLC technology also allow high-throughput analysis with enhanced sensitivity and separation efficiency (Chapter 14). On the other hand, the availability of monolithic columns has enabled the separation of small molecules with fast gradients and high flow rates (Chapters 9 and 11-13).

\section{"An excellent review about the theory, configuration and operation of multiplexing LC-MS systems is presented..."}

This book devotes a significant portion to covering the developments in multiplexing strategies to dramatically reduce cycle time for higher throughput. Multiple HPLC systems can be operated in parallel and share the same MS detector by staggering injections or adopting a multiplexed electrospray interface. An excellent review about the theory, configuration and operation of multiplexing LC-MS systems is presented in Chapter 4. Additional information on parallel chromatography can be found in Chapters 2, 3, 8, 11 and 12. It is also worth noting that recent advances in microfluidics and automation have resulted in the development of a novel microparallel LC system, which miniaturizes HPLC analysis by enabling 24 simultaneous separations and real-time UV and fluorescence detection. A detailed review of its instrumentation, performance and potential applications in the pharmaceutical industry is presented in Chapter 6. Alternatively, cassette dosing and sample pooling have been implemented to improve the throughput of drug-discovery pharmacokinetic screening (Chapters 7 and 12).

\footnotetext{
"There is no doubt ... that high-throughput analysis will continue to play an important role in drug discovery and development in the next $5-10$ years."
}

It was reported that $40 \%$ of failures in clinical trials were due to pharmacokinetic problems. Therefore, early drug metabolism and pharmacokinetics became critical in supporting drug discovery. Chapter 7 presents an excellent overview of strategies and techniques for high-throughput absorption, distribution, metabolism and excretion/pharmacokinetic analysis. It offers some useful guidance on how to make proper decisions in the screening process. Additional information regarding the analysis in drug metabolism during drug discovery is presented in Chapters 5 and 8 . Even though this book mainly focuses on the analysis of small molecules, it also includes a review on applying MS-based proteomics to study complex protein mixtures in Chapter 15. Also, with the enormous volume of data being generated by high-throughput analysis, a reliable, manageable and user-friendly informatics system is needed for reporting, storing and retrieving the data. This book only briefly mentions this subject in Chapter 8. A more complete review on the progress in this area would have been very helpful. Nevertheless, the importance and potential influence of this book are not to be affected. This book remains a quick and valuable resource for readers, ranging from professionals to academics, to gain knowledge about the most recent developments of high-throughput analysis in the pharmaceutical industry.

\section{Future perspective}

There is no doubt in my mind that highthroughput analysis will continue to play an important role in drug discovery and development in the next 5-10 years. Technological advances in the areas of sample preparation, chromatographic separation and MS detection are likely to further reduce the turnaround time and cost. For instance, much smaller sample size will be required for the detection of multiple analytes due to the application of microextraction and microfluidic techniques. Smaller sample size also allows multiple time points to be collected from a single animal (e.g., mouse) to reduce the subject-to-subject variability. Online sample-preparation approaches, which enable the direct injection of untreated biological samples, will gain more popularity. Multiple LC systems connected to a single MS for detector timesharing will be easier to set up and operate with greater flexibility. Also, integrated multidimensional chromatographic systems will offer the applications to a large variety of samples, due to their ability to effectively clean up impurities, 
salts and detergents, for example. On the other hand, with proper sample pretreatment, LC separation may possibly be eliminated and replaced by flow injection to an ESI source or analysis using MALDI. High-throughput analysis can also be increasingly important in the characterization and quantitation of proteins and peptides in biomarker discovery and development of biotherapeutics.

\section{Financial \& competing interests disclosure}

The author has no relevant affliations or financial involvement with any organization or entity with a financial interest in or financial conflict with the subject matter or materials discussed in the manuscript. This includes employment, consultancies, honoraria, stock ownership or options, expert testimony, grants or patents received or pending, or royalties.

No writing assistance was utilized in this manuscript. 